\title{
Understanding Non-Covalent Interactions: Correlated Energy Decomposition Analysis and Applications to Halogen Bonding
}

\author{
Jerome F. Gonthier ${ }^{\star \star a}$, Jonathan Thirman ${ }^{\mathrm{b}}$, and Martin Head-Gordon ${ }^{\mathrm{a}}$ \\ §SCS-Metrohm Award for best oral presentation in Computational Chemistry
}

\begin{abstract}
Non-covalent interactions play a primordial role in chemistry. Beyond their quantification, the detailed understanding of their physical processes is necessary to rationalize chemical trends and improve designs of chemical systems. Energy decomposition analyses allow detailed insight into non-covalent interactions by extracting electrostatics, Pauli repulsion, polarization, dispersion and charge transfer components from interaction energies. Recent work has demonstrated that electronic correlation influenced significantly all of these energy components, whereas previous decompositions only partitioned correlation between dispersion and charge transfer. The MP2 energy decomposition analysis with Absolutely Localized Molecular Orbitals (MP2 ALMO-EDA) takes these results fully into account and offers a correlation correction for each extracted component. A recent detailed investigation of the CCSD dispersion energy showed that a small number of virtual orbitals is sufficient to describe dispersion interactions accurately in the long-range, which potentially offers a basis-set independent definition of dispersion. Finally, we present an application of MP2 ALMO-EDA to a series of unusual halogen bonding complexes where charge transfer dominates over the electrostatic $\sigma$-hole interaction.
\end{abstract}

Keywords: Dispersion interactions · Electron correlation · Energy decomposition analysis · Halogen bonding · Non-covalent interactions

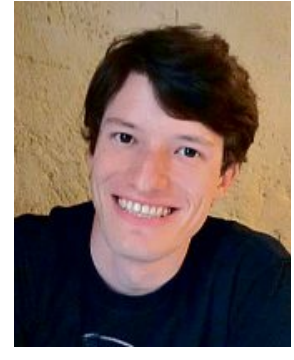

Jerome F. Gonthier studied chemistry at Ecole Polytechnique Federale de Lausanne (EPFL) in Switzerland. He conducted his Master thesis at the Max Planck Institute for Coal Research in Muhlheim an der Ruhr (Germany) under the supervision of Prof. Walter Thiel. He came

${ }^{*}$ Correspondence: Dr. J. F. Gonthiera E-mail: j.gonthier01@gmail.com

aKenneth S. Pitzer Center for Theoretical Chemistry Department of Chemistry, University of California

Berkeley, California, 94720 USA

and Chemical Sciences Division

Lawrence Berkeley National Laboratory

Berkeley, California, 94720 USA

${ }^{b}$ Argonne Leadership Computing Facility

Argonne National Laboratory, Argonne, IL 60439 USA back to EPFL to obtain his $\mathrm{PhD}$ in computational chemistry with Prof. Clemence Corminboeuf. He was then awarded an Early Post-Doc Mobility fellowship from the Swiss National Science Foundation to work with Prof. David Sherrill on Symmetry-Adapted Perturbation Theory in Atlanta (USA), after which he obtained an Advanced Post-Doc Mobility fellowship to join Prof. Martin Head-Gordon in University of California at Berkeley (USA), where he is currently working on understanding non-covalent interactions, notably through energy decomposition analysis methods and high-level computations.

\section{Introduction}

Non-covalent interactions play decisive roles in chemistry, determining for example the formation of pre-reactive complexes, ${ }^{[1]}$ the preferred orientation in enantioselective reactions, ${ }^{[2]}$ the conformations of biological[3] or supramolecular ${ }^{[4]}$ assemblies, or the most stable structure of molecular crystals. ${ }^{[5]}$ Nowadays, quantum chemistry methods exist to quantify noncovalent interactions accurately. However, a detailed understanding of their physical processes beyond their quantification is necessary to rationalize chemical trends in terms of practical concepts, our basis for understanding chemistry.

For this purpose, energy decomposition analysis (EDA) schemes were developed as early as the 1970s, when Kitaura and Morokuma[6] first decomposed the Hartree-Fock (HF) interaction energy into electrostatic, Pauli repulsion, polarization, and charge transfer components. The behavior of these original components was not completely satisfactory and they did not add up to the total interaction energy. Since then, many improved schemes have addressed these deficiencies, and some extended to Density Functional Theory (DFT) energies. ${ }^{[7]}$ We are particularly interested in this minireview in variational EDA schemes that obtain each energy component by fully optimizing a quantummechanically correct wavefunction with various constraints. The Absolutely Localized Molecular Orbitals EDA (ALMO-EDA) ${ }^{[7 \mathrm{~g}, 8]}$ enters this category and has recently seen some interesting developments regarding electronic correlation that we will review. Note that a closely related scheme is the Block-Localized Wavefunction EDA (BLW-EDA) ${ }^{[7 f]}$ and that both are based on the same technique 
to solve Hartree-Fock equations with localization constraints on the orbitals. [9]

To obtain consistent and accurate EDA components, it is desirable to design EDA schemes for post-HF methods such as MP2 or Coupled Cluster with Singles, Doubles and perturbative Triples, $\operatorname{CCSD}(\mathrm{T})$, the current golden standard of quantum chemistry. In particular, since decompositions of the Hartree-Fock contribution to intermolecular interactions are already well-studied, it is desirable to obtain a decomposition of the electronic correlation contribution, i.e. the correlation binding energy (CBE). Symmetry-Adapted Perturbation Theory $(\mathrm{SAPT})^{[10]}$ provides a solution through a perturbative expansion of the interaction energy that includes electron correlation, however SAPT relies on the assumption that the intermolecular interaction is weak. Variational EDA schemes such as ALMOEDA are applicable to the analysis of covalent bonds, ${ }^{[11]}$ and their variational nature even allowed recent work to incorporate geometric deformation effects in each energetic term. ${ }^{[12]}$

Previous variational EDAs only partitioned electronic correlation into dispersive or charge transfer energy components. Such decompositions have been applied by various groups to MP2 ${ }^{[13]}$ and to CCSD energies. ${ }^{[7 e, 14]}$ Here we will review recent developments in the understanding and decomposition of correlation binding energies, and demonstrate their utility in an example halogen bonding application.

\section{Influence of Electronic Correlation on Monomer Properties}

Since electron correlation lowers the total energy of a molecular system, we could intuitively expect that it would also lower intermolecular binding energies (i.e. enhance binding), essentially because electron correlation introduces attractive dispersion effects that are absent in Hartree-Fock wavefunctions.

This was disproven already in 1986 by Scheiner and coworkers ${ }^{[16]}$ who obtained repulsive correlation corrections to the water dimer binding energy. This early discovery seems however to have been known mostly in the SAPT community. ${ }^{[17]}$ Indeed, the first direct decompositions of CBE that appeared later only partitioned it in attractive terms. ${ }^{[73,13,14]}$ In 2014, Thirman et $a l .{ }^{[15]}$ reinvestigated this issue in detail for various conformers of the water dimer with different alignments of the monomer's dipoles (see Fig. 1). CBEs were computed at the RI-MP2 level for various monomer separations and at the CCSD(T) level for a O ... O separation of $5.5 \AA$ (see Fig. 2).

The CBEs for geometries having a favorable electrostatic interaction (equilib- rium (a) and aligned (b) on Fig. 1) become positive in the long and medium range, whereas they are always negative for geometries with vanishing (perpendicular, Fig. 1d) or repulsive (antialigned, Fig. 1c) dipole interactions. This result is valid for MP2 and $\operatorname{CCSD}(\mathrm{T})$, and is reproduced with several basis sets. ${ }^{[15]}$ The origin of repulsive correlation corrections was uncovered by examining the dipole moments of the monomers computed with $\mathrm{HF}$ and MP2. ${ }^{[15,16]}$ Indeed, HF tends to overestimate the dipole moments of molecules, which is corrected by the inclusion of electron correlation. Hence, electrostatic interactions in MP2 are less attractive than

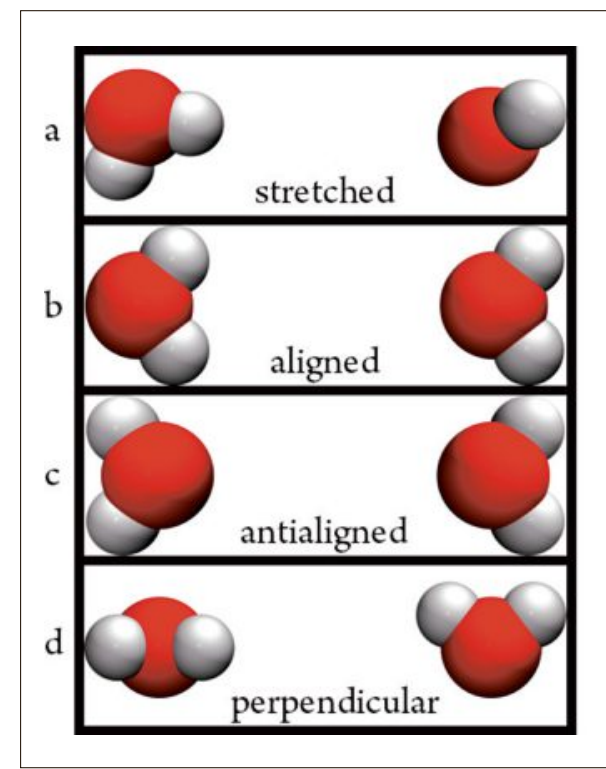

Fig. 1. Four different geometries of the water dimer. The stretched geometry (a) is based on the S22 equilibrium geometry. The other three geometries each have equivalent monomers with a bond length of $0.9584 \AA$ and a bond angle of $104.46 \circ$. Reprinted with permission from ref. [15]. Copyright 2014 American Chemical Society.

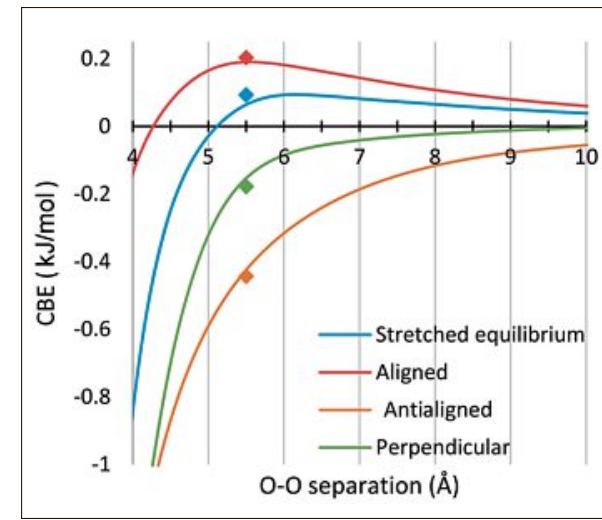

Fig. 2. RIMP2 correlation binding energy (CBE) curves for the water dimer in four different geometries, along with a $\operatorname{CCSD}(T)$ value at $5.5 \AA$ A O... O separation for each. Reprinted with permission from ref. [15]. Copyright 2014 American Chemical Society. those in HF because the electric moments of the interacting molecules generally become smaller.

This electrostatic-based rationalization of the CBE is consistent with the above observations for the various water conformers (see Fig. 2). The equilibrium and aligned conformers have attractive dipole-dipole interactions, which are overestimated at the Hartree-Fock level, and thus their CBE is positive. The perpendicular conformer has a vanishing dipole-dipole interaction and thus its correlation correction essentially represents dispersion, quickly decaying to zero. Finally, the anti-aligned dimer has repulsive dipole-dipole interactions, once again overestimated by Hartree-Fock, so in this case the CBE is negative since it reduces the repulsion between the monomers. ${ }^{[15]}$

Electronic correlation affects significantly molecular properties, including electric moments and polarizability. As a consequence, correlation binding energies do not only contain attractive components, as has been previously assumed in EDAs. $[7 e, 13,14]$ In the next section, we will summarize MP2 ALMO-EDA, which was built on this knowledge.

\section{MP2 ALMO-EDA}

\subsection{Theory}

The HF ALMO-EDA method relies on intermediate constrained wavefunctions to separate the various physical components of an intermolecular interaction energy. In MP2 ALMO-EDA, an MP2 correction is computed on top of each intermediate HF wavefunction. We here present each intermediate briefly, full details of the theory ${ }^{[18]}$ and of the implementation ${ }^{[19]}$ being available elsewhere:

1. Computing MP2 on isolated monomers, saving the HF orbitals and the MP2 amplitudes $t_{i j}{ }^{a b}$ from which the MP2 energy is computed.

2. Allowing the monomers to interact while keeping their orbitals and MP2 amplitudes frozen. MP2 is non-variational, thus the corresponding energy correction is based on the Hylleraas functional and includes orbital relaxation to yield the correct monomer MP2 dipole moments.

3. Polarizing wavefunctions in the field of their interacting partner. Absolutely Localized MolecularOrbitals(ALMOs) are optimized on each monomer using only basis functions on the same monomer to prevent charge delocalization. The MP2 correction is computed including only excitations on the same monomer (top row of Fig. 3).

4. Adding all charge-conserving correlations (two first rows of Fig. 3) in the 
MP2 correction. These include simultaneous excitations on both monomers associated with dispersion.

5. Computing the full MP2 energy of the dimer, including fully relaxed $\mathrm{HF}$ orbitals and all excitations in Fig. 3.

In each step, we allow an additional physical effect to influence the wavefunction. Thus the frozen energy is obtained as the energy difference between steps 1 and 2 above, the polarization energy as the difference between steps 2 and 3, the dispersion energy as the difference between steps 3 and 4 and the charge transfer energy as the difference between steps 4 and 5. Each contains a correlation correction from MP2. MP2 ALMO-EDA was implemented in a locally modified version of Q-Chem ${ }^{[20]}$ and will be released soon.

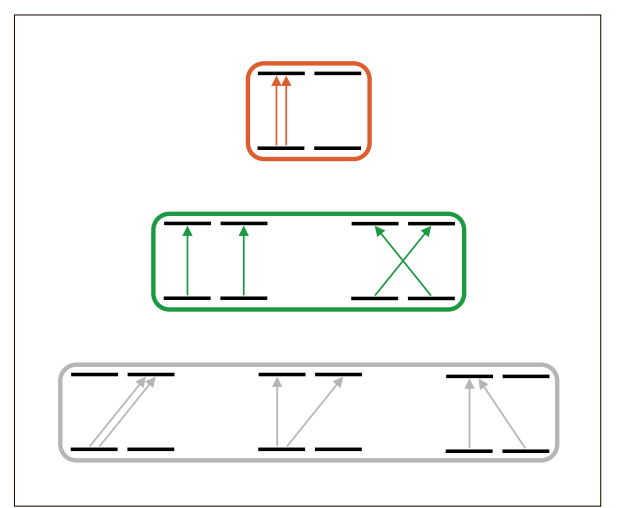

Fig. 3. The three types of double excitations in MP2: on monomer (upper row), charge conserving (middle row), charge transferring (bottom row). Reprinted from ref. [18], with the permission from AIP Publishing.

\subsection{Basis Set Limit Behavior}

The polarized wavefunctions in step 3 are defined by ALMOs which rely on the locality of atom-centered basis functions on each monomer. However, in large basis sets, basis functions centered on one monomer will overlap with the other monomer. As a result, the ALMO orbitals will partially delocalize over both monomers, and the polarized wavefunctions will include some charge transfer contamination. In the complete basis set (CBS) limit, the polarization energy will contain $100 \%$ of the charge transfer effect and the charge transfer energy will vanish.

Recently, Horn and Head-Gordon ${ }^{[21]}$ proposed a solution: constraining the polarized wavefunction to relax in a virtual space specifically tailored to represent only polarization. This virtual space is built from Fragment Electric Field Response Functions (FERFs), which exactly represent the deformation of the occupied orbitals of one monomer subjected to an external static electric field. Thus, the FERFs naturally exclude charge transfer even in the complete basis set limit.
A related issue appears in MP2 ALMOEDA in the definition of the dispersion energy. In Fig. 3, we discriminate between charge-conserving and charge-transferring excitations based on the localization of the ALMOs on one or the other monomer. As the basis set is extended towards the CBS, excitations classified as charge-conserving will delocalize more and more over the partner monomer until they fully represent the MP2 charge transfer contribution. Similarly to the FERFs for polarization, it is then desirable to obtain a virtual space specifically tailored to represent dispersion interactions. To understand how to design it, we investigated compressed representations of the virtual space for CCSD dispersion energies.

\section{Dispersion-specific Virtual Orbitals}

\subsection{Compact Representation of Dispersion}

To investigate the compactness of the virtual space for dispersion energies and extract the corresponding virtuals, we examined the CCSD dispersion energy in detail. ${ }^{[22]}$ Our investigation was limited to the long-range limit of dispersion energy $E^{\text {disp }}$, where the overlap between the monomers is negligible and dispersion well-defined:

$$
E_{C C S D}^{d i s p}=\sum_{i a}^{A} \sum_{j b}^{B} t_{i j}^{a b}\left(\phi_{a} \phi_{i} \mid \phi_{b} \phi_{j}\right)
$$

where $i, a$ are respectively occupied and virtual orbital indices localized on monomer A while $j, b$ are respectively occupied and virtual orbital indices localized on monomer B. We will denote the set of amplitudes $t_{i j}^{a b}$ for which the indices satisfy the above $T_{2}^{\text {disp }}$ and refer to them as dispersion amplitudes. They are easily extracted from a CCSD computation performed in a localized orbital basis.

We extracted the most important components of $T_{2}^{\text {disp }}$ using Singular Value Decomposition (SVD). SVD factorizes a matrix as a set of right and left singular vectors whose contributions to the original matrix are ranked by singular values. To

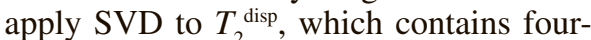
indices amplitudes $t_{i j}{ }^{a b}$, we arranged the amplitudes in a large matrix with indices $i$ and $a$ on the rows and indices $j$ and $b$ on the columns. This groups together indices belonging to the same monomers, which yields singular vectors that are reflective of monomer's properties. Each of these singular vectors contains both occupied and virtual indices, thus we will refer to them as geminal functions (i.e. functions of two electrons).
We performed this analysis for the $\mathrm{He}$ dimer at 3.0, 6.0 and 9.0 A separation with the doubly augmented d-aug-cc-pVQZ basis set. ${ }^{[23]}$ The obtained singular values are displayed in Fig. 4 on a logarithmic scale. Strikingly, the singular values decrease extremely rapidly, and this decrease becomes even more pronounced as the interatomic distance is increased. Closer inspection actually revealed that the three first singular values clearly dominate the other ones. Since this reflects the importance of the corresponding geminals in the original $T_{2}^{\text {disp }}$, a very small number of them should describe the amplitudes correctly. These conclusions held for a variety of two-electron systems and basis sets, ${ }^{[22}$ and remained valid for larger dimers up to the benzene-methane dimer.

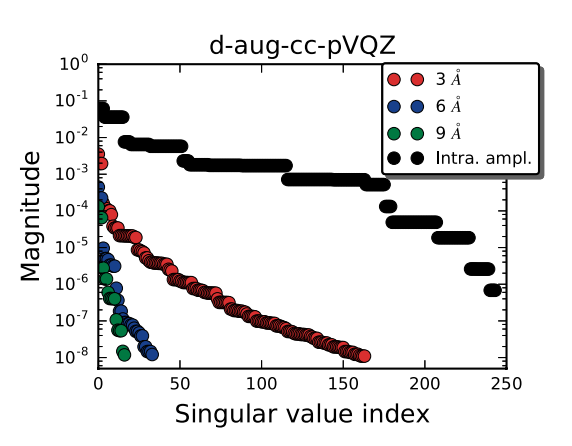

Fig. 4. CCSD $T_{2}^{\text {disp }}$ singular values for $\mathrm{He}_{2}$ at 3.0 (red), 6.0 (blue) and 9.0 (green) $\AA$ interatomic distances. In black, we show singular values obtained for the intraatomic amplitudes in $\mathrm{He}$, reflecting short-range electron correlation.

For comparison, Fig. 4 shows in black singular values from the same analysis performed for intraatomic amplitudes $\mathrm{t}_{\mathrm{ij}}{ }_{\mathrm{ab}}$ (where all indices belong to the same monomer) that describe short-range correlation. Clearly, the decay of intraatomic singular values is comparatively very slow. This reveals the qualitative difference between short-range and long-range correlation, and offers physical ground for using different approximations for both.

The approximate reconstruction of $T_{2}^{\text {disp }}$ from a limited number of geminals yielded good approximate dispersion energies. In all cases, we recovered between 85 and $98 \%$ of the full dispersion energy at short distance, to more than $99 \%$ at around 6.0 $\AA$ intermonomer separation and beyond. For the largest system investigated (benzene-methane dimer with aug-cc-pVTZ) the short-range relative percent error with only three geminals is about $15 \%$ of the full dispersion energy (Fig. 5). Increasing the number of geminals to reconstruct $T_{2}^{\text {disp }}$ quickly reduces the observed error. Upon increasing the separation between the monomers by $3.0 \AA$, the relative percent error also decreases dramatically, and using 


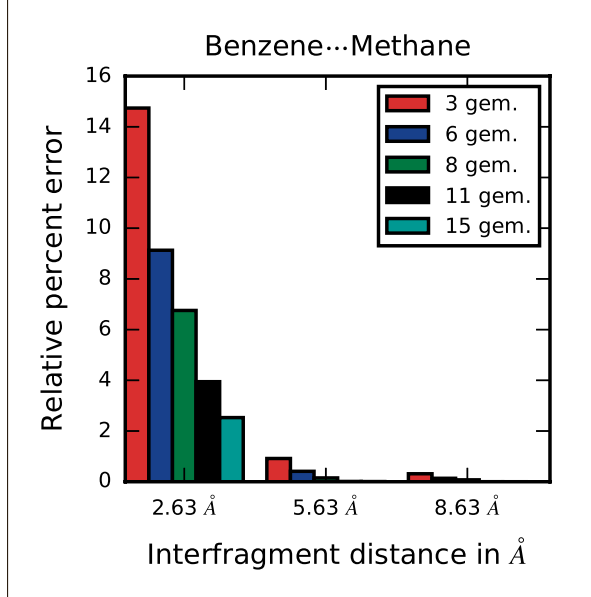

Fig. 5. Relative percent error for CCSD dispersion energy computed with $T_{2}$ disp approximately reconstructed with 3 (red), 6 (blue), 8 (green), 11 (black) and 15 (teal) geminals at various distances for the benzene-methane dimer with the aug-cc-pVTZ basis set.

only three geminals is already sufficient to reach $99 \%$ accuracy. These observations also held for $\mathrm{Ne}_{2},\left(\mathrm{CH}_{4}\right)_{2},\left(\mathrm{C}_{2} \mathrm{H}_{4}\right)_{2}$, and various two-electron systems. ${ }^{[22]}$

\subsection{Virtual Space}

Since only a few geminals describe both the dispersion amplitudes $T_{2}^{\text {disp }}$ and the dispersion energy, there must exist a compact virtual space associated with them. We again leveraged SVD to examine these virtual orbitals. We wrote each geminal as a matrix, with occupied orbital indices on the rows and virtual orbital indices on the columns. In this manner, the SVD right singular vectors represent new orbitals that compactly describe the virtual part of each geminal. For ease of visualization, we examined monomers with only one occupied orbital. In this case, there is only one virtual orbital per geminal, plotted for one monomer of $\mathrm{He}_{2}$ in Fig. 6.

In all two-electron systems in the longrange (6.0 $\AA$ and beyond), we observe the same shape of the virtual orbitals. The first three, corresponding to the three dominating geminals, are $2 p$-like orbitals. Since the occupied orbital is a $1 s$ function, these three geminals represent $1 s$ to $2 p$ excitations that generate a dipole moment. Thus, we connect these virtuals with the common description of dispersion as the interaction of instantaneous dipoles. The three next virtuals are $3 p$-like orbitals, and the five following them are $3 d$-like orbitals. We connect these two groups respectively to dipole-quadrupole and quadrupolequadrupole interactions in the multipolar expansion of dispersion. The distance dependence of the associated singular values corroborates this connection. ${ }^{[22]}$

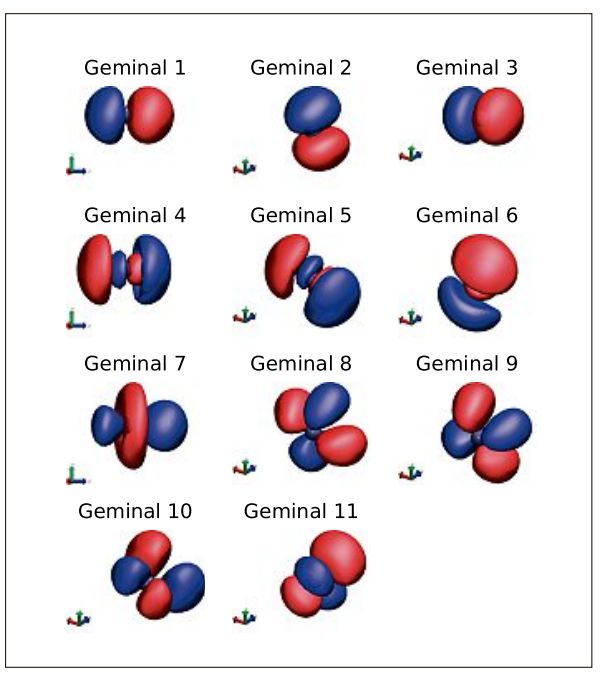

Fig. 6. Virtual part of geminals 1 to 11 for $\mathrm{He}_{2}$ with the d-aug-cc-pVQZ basis set at $6.0 \AA$ interatomic separation. Orbitals are plotted at an isovalue of 0.05 and the interacting partner is located along the z-axis, represented in blue. Reprinted from ref. [22], with the permission of AIP Publishing.

Our work demonstrated that there indeed exists a compact basis of virtual orbitals that efficiently represents dispersion energies, using only three functions per occupied orbital in the long range. This is in agreement with recent results obtained in the context of the Pair Natural Orbital (PNO) method, where it was numerically shown that only 3 to 4 virtual PNOs were needed to describe each long-range correlated electron pair. ${ }^{[24]}$ A virtual space specifically tailored for dispersion thus exists, which opens the way to a basis set independent definition of dispersion. We are currently exploring practical ways to extract the appropriate virtual space, using the CCSD-derived virtual orbitals as reference.

\section{Application to Halogen Bonding}

Halogen bonding is an attractive interaction involving a halogen atom $\mathrm{X}$ accepting a nucleophilic partner B, usually repre- sented as RX...B. These interactions have been known for a long time ${ }^{25]}$ and have gained increasing importance in recent years as a controllable factor to build molecular assemblies. ${ }^{[4,5,26]}$ Halogen bonds have originally been rationalized in terms of charge transfer between the interacting partners. ${ }^{[27]}$ However, another explanation appeared in terms of the $\sigma$-hole ${ }^{[28]}$ on the halogen atom, that is a region of positive electrostatic potential opposing the $\sigma$ bond with the rest of the molecule. The strength of halogen bonding interactions generally correlates with the size of the $\sigma$-hole.

Recently, Huber et al. ${ }^{[29]}$ uncovered a series of $\mathrm{CX}_{3} \mathrm{I} \cdots \mathrm{Y}$ complexes $(\mathrm{X}=\mathrm{F}, \mathrm{Cl}$, $\mathrm{Br}$, I and $\mathrm{Y}=$ halide ion or trimethylamine). In these complexes, the binding strength is inversely correlated with the size of the $\sigma$-hole. $X=F$ is the most electronegative substituent, that generates the strongest $\sigma$-hole and electrostatic interaction, while $\mathrm{CI}_{4}$ has zero dipole and the smallest $\sigma$-hole, yet exhibits the strongest interaction. To uncover the relative importance of electrostatics and charge transfer in these systems, Thirman and Head-Gordon ${ }^{[30]}$ applied MP2 ALMO-EDA to $\mathrm{CF}_{3} \mathrm{I}_{\cdots} \mathrm{F}^{-}$and $\mathrm{CI}_{4} \cdots \mathrm{F}^{-}$. Since the equilibrium distance in these two complexes differs (2.20 and $2.11 \AA$ respectively), a scan of $\mathrm{I}^{2} \cdot \mathrm{F}^{-}$distances was performed. For each distance, all other geometrical parameters were relaxed. The MP2 interaction energy (see original paper ${ }^{[30]}$ for full details) for each of these geometries is then decomposed to yield the energy components presented in Fig. 7 as a function of distance.

These curves show that $\mathrm{CI}_{4}$ exhibits larger charge transfer interactions (by about $20 \mathrm{~kJ} / \mathrm{mol}$ ) than $\mathrm{CF}_{3} \mathrm{I}$ at all distances. This is expected as the charge transfer tends to increase going down the periodic table. $\mathrm{CF}_{3}$ I possesses a higher dipole moment and a larger $\sigma$-hole, generating an important frozen energy term (that contains both electrostatics and Pauli repulsion). $\mathrm{CI}_{4}$ makes up for this difference by being more polarizable, and together the frozen and polarization terms make the two complexes bind with roughly the

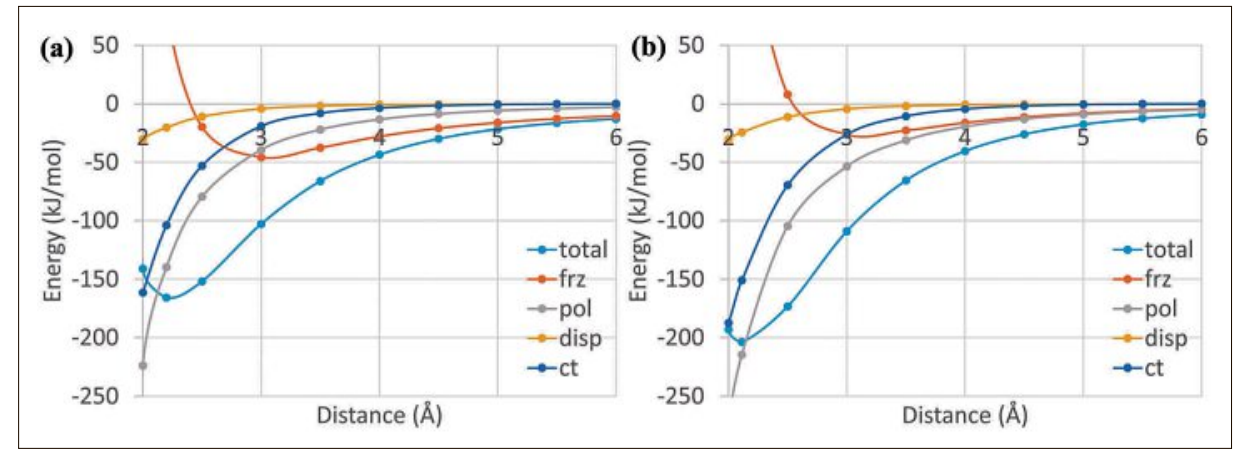

Fig. 7. MP2/def2-TZVPP interaction energy components for $\mathrm{CF}_{3} \mid \cdots \mathrm{F}^{-}(\mathrm{a})$ and $\mathrm{Cl}_{4} \cdots \mathrm{F}^{-}$(b). ${ }^{[30]}$ Reproduced by permission of the PCCP Owner Societies. 
same strength. We consider the frozen and polarization terms together for two reasons: they are both electrostatic in nature and linked to the $\sigma$-hole rationalization of halogen bonds; they both contain an orbital deformation contribution originating in the Pauli repulsion at short distance.

The difference in charge transfer energies only makes up for about half of the total difference in binding energies. Much of the rest comes from the closer equilibrium distance in $\mathrm{CI}_{4}$ compared to $\mathrm{CF}_{3} \mathrm{I}$, allowing a more favorable interaction. In addition, the geometric deformation energies to get from the isolated monomer to the equilibrium structure are $12 \mathrm{~kJ} / \mathrm{mol}$ for $\mathrm{CI}_{4}$ and $20 \mathrm{~kJ} / \mathrm{mol}$ for $\mathrm{CF}_{3} \mathrm{I}$. Thus, $\mathrm{CI}_{4}$ achieves a larger binding energy because of its large charge transfer component and because it is easily deformable into its dimer equilibrium geometry. The larger dipole moment and electrostatic interactions of $\mathrm{CF}_{3} \mathrm{I}$ are compensated by the larger polarizability of $\mathrm{CI}_{4}$.

Another perspective on this interaction is provided by the adiabatic MP2 ALMOEDA scheme.[12,30] Here, the geometry of the dimer is fully optimized for each energy component, allowing us to directly measure the effect of each type of interaction on the geometry. The influence of charge transfer is confirmed to be larger for $\mathrm{CI}_{4}$. The dimer geometries including only the frozen and the polarization contributions have similar interaction energies, corroborating the above interpretation that the larger polarization of $\mathrm{CI}_{4}$ compensates for the larger electrostatic interactions of $\mathrm{CF}_{3} \mathrm{I}$.

The adiabatic MP2 ALMO-EDA shows a shortening of the $\mathrm{C}-\mathrm{I}$ bond in $\mathrm{CI}_{4}$ when only frozen interactions are included, while charge transfer elongates the bond with respect to the equilibrium geometry. This means that, in the dimer equilibrium geometry, the dipole of $\mathrm{CI}_{4}$ opposes the interaction, whereas the dipole of $\mathrm{CF}_{3} \mathrm{I}$ is enhanced by the interaction. This highlights the fundamental difference between these two halogen bonds: $\mathrm{CI}_{4} \cdots \mathrm{F}^{-}$is driven both electronically and geometrically by charge transfer, overriding the electrostatic interactions, while $\mathrm{CF}_{3} \mathrm{I}_{\cdots} \mathrm{F}^{-}$is driven by electrostatic interactions, including the $\sigma$-hole on I.

As an example of a system that combines strong charge transfer with strong electrostatics, Thirman et al. then examined $\mathrm{C}\left(\mathrm{NO}_{2}\right)_{3} \mathrm{I} \cdots \mathrm{F}^{-}$. We reproduce the curves of the various energy components as a function of distance in Fig. 8. The binding energy is considerable at about $260 \mathrm{~kJ} / \mathrm{mol}$, which arises in part from the very strong dipole of $3.2 \mathrm{D}$ that generates electrostatic interactions more favorable than even $\mathrm{CF}_{3} \mathrm{I}$. The charge transfer energy is very important as well, being even slightly more favorable than in $\mathrm{CI}_{4}$. The

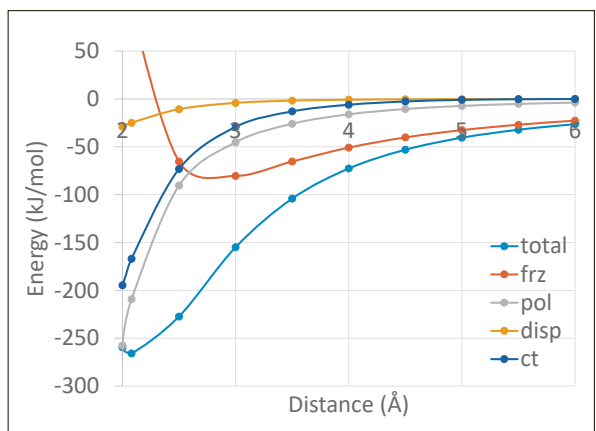

Fig. 8. MP2/def2-TZVPP interaction energy components for $\mathrm{C}\left(\mathrm{NO}_{2}\right)_{3} \mid \cdots \mathrm{F}^{-}$. $^{[30]}$ Reproduced by permission of the PCCP Owner Societies

polarizability of $\mathrm{C}\left(\mathrm{NO}_{2}\right)_{3} \mathrm{I}$ is in between those of $\mathrm{CF}_{3} \mathrm{I}$ and $\mathrm{CI}_{4}$. Thus, without the contribution of charge transfer it would be difficult to predict the considerable binding energy observed.

Thanks to accurate energy components provided by MP2 ALMO-EDA, the three systems investigated demonstrate that both electrostatics and charge transfer can play major roles in determining the strength of halogen bonds, in agreement with recent findings on $\lambda^{3}$-iodane complexes. ${ }^{[31]}$ Moreover, they illustrate a case where polarizability and charge transfer follow different trends, which emphasizes the importance of discriminating between these two components.

\section{Conclusions}

The development of energy decompositions for correlated, accurate methods benefits from careful analyses of the correlation contribution to non-covalent interaction energies and from detailed investigations of each interaction component. Future work will focus on designing practical ways to obtain a compact dispersionspecific virtual space for the MP2 ALMOEDA scheme. The exceptional compressibility of the virtual space for long-range correlation may find applications in local methods, as an alternative to the recent work by Werner ${ }^{[24]}$ on reduced Pair Natural Orbital space for dispersive interactions.

\section{Acknowledgments}

This work was supported by the U.S. National Science Foundation through Grant CHE-1665315, with additional support for J.T. from the U.C. Berkeley CalSolv Center. J.F.G. acknowledges Grant No. P300P2_164631 from the Swiss National Science Foundation.

Received: February 28, 2018
[1] A. J. Neel, M. J. Hilton, M. S. Sigman, F. D. Toste, Nature 2017, 543, 637.

[2] a) L. M. Salonen, M. Ellermann, F. Diederich, Angew. Chem. Int. Ed. 2011, 50, 4808; b) N. Vallavoju, J. Sivaguru, Chem. Soc. Rev. 2014, 43, 4084; c) H. J. Davis, R. J. Phipps, Chem. Sci. 2017, 8, 864.

[3] Y. Fukunishi, in 'OMICS', CRC Press, 2011, p. 281.

[4] L. C. Gilday, S. W. Robinson, T. A. Barendt, M. J. Langton, B. R. Mullaney, P. D. Beer, Chem. Rev. 2015, 115, 7118.

[5] F. Meyer, P. Dubois, CrystEng Comm 2013, 15, 3058.

[6] a) K. Morokuma, J. Chem. Phys. 1971, 55, 1236; b) K. Kitaura, K. Morokuma, Int. J. Quantum Chem. 1976, 10, 325.

[7] a) G. Frenking, K. Wichmann, N. Frohlich, C Loschen, M. Lein, J. Frunzke, V. M. Rayon, Coord. Chem. Rev. 2003, 238-239, 55; b) T. Ziegler, A. Rauk, Theor. Chem. Acc. 1977, 46, $1 ;$ c) K. A. Olszewski, M. Gutowski, L. Piela, $J$. Phys. Chem. 1990, 94, 5710; d) P. S. Bagus, K. Hermann, J. Bauschlicher, W. Charles J. Chem. Phys. 1984, 80, 4378; e) P. Su, H. Li, J. Chem Phys. 2009, 131, 014102; f) Y. Mo, J. Gao, S. D. Peyerimhoff, J. Chem. Phys. 2000, 112, 5530; g) R. Z. Khaliullin, E. A. Cobar, R. C. Lochan, A. T. Bell, M. Head-Gordon, J. Phys. Chem. A 2007, 111, 8753 .

[8] P. R. Horn, Y. Mao, M. Head-Gordon, Phys Chem. Chem. Phys. 2016, 18, 23067.

[9] a) R. Z. Khaliullin, M. Head-Gordon, A. T. Bell, J. Chem. Phys. 2006, 124, 204105; b) H. Stoll, G. Wagenblast, H. Preuss, Theor. Chem. Acc. 1980, 57, 169; c) E. Gianinetti, M. Raimondi, E. Tornaghi, Int. J. Quantum Chem. 1996, 60 , 157.

[10] a) B. Jeziorski, R. Moszynski, K. Szalewicz, Chem. Rev. 1994, 94, 1887; b) K. Szalewicz, WIREs Comput. Mol. Sci. 2012, 2, 254.

[11] a) D. S. Levine, M. Head-Gordon, PNAS, Early Ed. 2017, doi.org/10.1073/pnas.171563114; b) D. S. Levine, P. R. Horn, Y. Mao, M. HeadGordon, J. Chem. Theory Comput. 2016, 12, 4812.

[12] Y. Mao, P. R. Horn, M. Head-Gordon, Phys Chem. Chem. Phys. 2017, 19, 5944.

[13] a) J. Langlet, J. Caillet, J. Berges, P. Reinhardt, J. Chem. Phys. 2003, 118, 6157; b) S. Grimme, C. Muck-Lichtenfeld, J. Antony, Phys. Chem. Chem. Phys. 2008, 10, 3327; c) M. Schutz, G. Rauhut, H.-J. Werner, J. Phys. Chem. A 1998 , 102, 5997.

[14] a) R. J. Azar, M. Head-Gordon, J. Chem. Phys. 2012, 136, 024103; b) W. B. Schneider, G. Bistoni, M. Sparta, M. Saitow, C. Riplinger, A. A. Auer, F. Neese, J. Chem. Theory Comput. 2016, 12, 4778; c) D. G. Fedorov, K. Kitaura, $J$. Comput. Chem. 2007, 28, 222.

[15] J. Thirman, M. Head-Gordon, J. Phys. Chem. Lett. 2014, 5, 1380.

[16] M. M. Szczesniak, S. Scheiner, J. Chem. Phys. 1986, 84, 6328.

[17] a) M. M. Szczesniak, R. J. Brenstein, S. M. Cybulski, S. Scheiner, J. Phys. Chem. 1990, 94 , 1781; b) P. Hobza, R. Zahradnik, Chem. Rev. 1988, 88, 871; c) K. Szalewicz, S. J. Cole, W. Kolos, R. J. Bartlett, J. Chem. Phys. 1988, 89 , 3662; d) S. Rybak, B. Jeziorski, K. Szalewicz, J. Chem. Phys. 1991, 95, 6576.

[18] J. Thirman, M. Head-Gordon, J. Chem. Phys. $\mathbf{2 0 1 5}, 143,084124$

[19] J. Thirman, M. Head-Gordon, J. Phys. Chem. A 2017, 121, 717 .

[20] Y. Shao, Z. Gan, E. Epifanovsky, A. T. B. Gilbert, M. Wormit, J. Kussmann, A. W. Lange, A. Behn, J. Deng, X. Feng, D. Ghosh, M. Goldey, P. R. Horn, L. D. Jacobson, I. Kaliman, R. Z. Khaliullin, T. Kus, A. Landau, J. Liu, E. I. Proynov, Y. M. Rhee, R. M. Richard, M. A. 
Rohrdanz, R. P. Steele, E. J. Sundstrom, I. I. I. H Lee Woodcock, P. M. Zimmerman, D. Zuev, B. Albrecht, E. Alguire, B. Austin, G. J. O. Beran, Y. A. Bernard, E. Berquist, K. Brandhorst, K. B. Bravaya, S. T. Brown, D. Casanova, C.-M. Chang, Y. Chen, S. H. Chien, K. D. Closser D. L. Crittenden, M. Diedenhofen, R. A. D. Jr., H. Do, A. D. Dutoi, R. G. Edgar, S. Fatehi L. Fusti-Molnar, A. Ghysels, A. GolubevaZadorozhnaya, J. Gomes, M. W. D. HansonHeine, P. H. P. Harbach, A. W. Hauser, E. G. Hohenstein, Z. C. Holden, T.-C. Jagau, H. Ji, B. Kaduk, K. Khistyaev, J. Kim, J. Kim, R. A. King, P. Klunzinger, D. Kosenkov, T. Kowalczyk, C. M. Krauter, K. U. Lao, A. D. Laurent, K. V. Lawler, S. V. Levchenko, C. Y. Lin, F. Liu, E. Livshits, R. C. Lochan, A. Luenser, P. Manohar, S. F. Manzer, S.-P. Mao, N. Mardirossian, A. V. Marenich, S. A. Maurer, N. J. Mayhall, E. Neuscamman, C. M. Oana, R. Olivares-Amaya, D. P. O’Neill, J. A. Parkhill, T. M. Perrine, R. Peverati, A. Prociuk, D. R. Rehn, E. Rosta, N. J. Russ, S. M. Sharada, S. Sharma, D. W. Small, A. Sodt, T. Stein, D. Stuck, Y.-C. Su, A. J. W. Thom, T. Tsuchimochi,
V. Vanovschi, L. Vogt, O. Vydrov, T. Wang, M. A. Watson, J. Wenzel, A. White, C. F. Williams, J. Yang, S. Yeganeh, S. R. Yost, Z.-Q. You, I Y. Zhang, X. Zhang, Y. Zhao, B. R. Brooks, G. K. L. Chan, D. M. Chipman, C. J. Cramer, I. I. I. William A. Goddard, M. S. Gordon, W. J. Hehre, A. Klamt, I. I. I. Henry F. Schaefer, M. W. Schmidt, C. D. Sherrill, D. G. Truhlar, A. Warshel, X. Xu, A. Aspuru-Guzik, R. Baer, A. T. Bell, N. A. Besley, J.-D. Chai, A. Dreuw, B. D. Dunietz, T. R. Furlani, S. R. Gwaltney, C.-P. Hsu, Y. Jung, J. Kong, D. S. Lambrecht, W. Liang, C. Ochsenfeld, V. A. Rassolov, L. V. Slipchenko, J. E. Subotnik, T. V. Voorhis, J. M Herbert, A. I. Krylov, P. M. W. Gill, M. HeadGordon, Mol. Phys. 2015, 113, 184.

[21] P. R. Horn, M. Head-Gordon, J. Chem. Phys 2015, 143, 114111.

[22] J. F. Gonthier, M. Head-Gordon, J. Chem. Phys. 2017, 147, 144110.

[23] a) D. E. Woon, T. H. Dunning, J. Chem. Phys 1994, 100, 2975; b) R. A. Kendall, T. H Dunning, R. J. Harrison, J. Chem. Phys. 1992 96, 6796; c) T. H. Dunning, Jr., J. Chem. Phys. 1989, 90, 1007 .
[24] H.-J. Werner, J. Chem. Phys. 2016, 145, 201101.

[25] F. Guthrie, J. Chem. Soc. 1863, 16, 239.

[26] a) P. Metrangolo, H. Neukirch, T. Pilati, G. Resnati, Acc. Chem. Res. 2005, 38, 386; b) G. Cavallo, P. Metrangolo, R. Milani, T. Pilati, A. Priimagi, G. Resnati, G. Terraneo, Chem. Rev. 2016, 116, 2478.

[27] a) O. Hassel, Science 1970, 170, 497; b) A. E. Reed, F. Weinhold, R. Weiss, J. Macheleid, J. Phys. Chem. 1985, 89, 2688.

[28] T. Clark, M. Hennemann, J. S. Murray, P. Politzer, J. Mol. Model. 2007, 13, 291.

[29] S. M. Huber, E. Jimenez-Izal, J. M. Ugalde, I. Infante, Chem. Commun. 2012, 48, 7708.

[30] J. Thirman, E. Engelage, S. Huber, M. HeadGordon, Phys. Chem. Chem. Phys. 2018, 20, 905.

[31] H. Pinto de Magalhães, A. Togni, H. P. Lüthi, $J$. Org. Chem. 2017, 82, 11799. 